\title{
THERMAL MODEL DEVELOPMENT FOR A CUBESAT
}

\author{
Nawar Al Hemeary ${ }^{* 1,2}$, Maciej JaWorsk ${ }^{3}$, Jan Kindrack ${ }^{3}$, and Gábor SzederkényI ${ }^{1}$ \\ ${ }^{1}$ Faculty of Information Technology and Bionics, Pázmány Péter Catholic University, Práter u. 50/A, \\ Budapest, 1083, HUNGARY \\ ${ }^{2}$ Electromechanical Engineering Department, University of Technology, Al Senaha St., Baghdad, 10066, \\ IRAQ \\ ${ }^{3}$ Faculty of Power and Aeronautical Engineering, Warsaw University of Technology, Nowowiejska 24, \\ Warszawa, 00-665, POLAND
}

\begin{abstract}
CubeSats provide a cost-effective means of several functions of satellites due to their small size, mass, relative simplicity and short development time. Therefore, CubeSat technologies have been widely studied and developed by space organizations, companies and educational institutions all over the world. These satellites have certain drawbacks. Small surface areas are a consequence of their small size which often imply thermal and power constraints. A novel development of CubeSats known as PW-Sat has been developed by Warsaw University of Technology. A control-oriented lumped thermal model of this satellite containing a fuel tank in the form of nonlinear ordinary differential equations is proposed in this paper. The model is able to simulate the thermal behavior of the surface and fuel tank of the satellite in its orbit. For the PW-Sat to operate reliably, the temperature of the fuel tank has to be maintained within given safety limits. Because of the limited power, passive thermal control is assumed in this case. Several simulation results are presented for different surface compositions to determine whether they are able to guarantee the prescribed temperature range throughout the entire orbit or not.
\end{abstract}

Keywords: PW-Sat, TMM, thermal behavior, propellant tank, dynamical modeling

\section{INTRODUCTION}

In recent years, interest in Cube-Satellites (CubeSats) has grown tremendously within the space community from space agencies as well as in industry and academia. Two factors have influenced this spurt of interest, namely the low-cost nature of access to space and the utilization of commercial off-the-shelf (COTS) technologies in the design architecture. These two factors have led to a significantly low overall cost of a CubeSat mission [1]. A CubeSat is cubic in form with edges $10 \mathrm{~cm}$ in length and a mass of up to $1.33 \mathrm{~kg}$. The PW-Sat is a CubeSat that has been in development for more than a year by different teams at Warsaw University of Technology [2,3]. At present, PW-Sat has never been flown with an onboard propulsion system. Due to the significant and growing interest in CubeSat mission capabilities, several propulsion systems have been rapidly developed for use in CubeSats such as cold gas propulsion systems, solar sails, electric propulsion systems and chemical propulsion systems $[4,5]$. Cold gas propulsion systems are relatively simple solutions in CubeSats. Gas from a high-pressure gas cylinder is simply vented through a valve and nozzle to produce thrust $[6,7]$.

\footnotetext{
*Correspondence: al.hemeary@itk.ppke.hu
}

The goal of this paper is to propose a lumped dynamical model of temperatures during orbital motion in the main parts of a CubeSat that contains a fuel tank. The model is built in the form of nonlinear ordinary differential equations (ODEs). Although advanced thermal simulation tools exist that utilize detailed distributed mathematical models, this simple form of a model has been chosen since it is intended to be used in the model of temperature control design. The overwhelming majority of control design techniques require models in the form of ordinary differential equations [8]. Moreover, in the case of nonlinear models such as the CubeSat system studied, a low dimensional model is preferred due to the computational complexity of control design. This approach is also supported by control theory and practice, namely that in general such simple models are sufficient for controller design [9]. As a first step in terms of the regulation of temperature, passive control in the form of the appropriate composition of materials covering the surfaces of the satellite is used. During the construction of the model, the standard principles of thermal modeling $[10,11]$ and their application in aerospace engineering are followed $[12,13]$.

Relevant results can be found in the literature concerning the thermal modeling and analysis of small satel- 
lites in the form of ODEs. In [14] a simple thermal dynamical model of a CubeSat containing two differential equations is presented. The two lumped balance volumes are the surface and internal parts of the satellite, respectively. It is shown that the problem is mathematically analogous to the forced vibration of a damped mechanical system. In [9], new theoretical results on the qualitative behavior of spacecraft thermal models are provided that contain several nodes (compartments). It is proven that such models exhibit a unique asymptotically stable equilibrium in the positive orthant with constant external disturbance inputs which leads to a stable limit cycle during orbital motion. The analysis concerning the frequency domain of a multi-compartmental model of a satellite is conducted in [15]. The ODEs are linearized around the equilibrium points which permit the use of Fourier analysis.

The structure of the paper is as follows: the description starts with the derivation of a simple mathematical model to simulate the transient thermal behavior of the fuel tank as well as the satellite faces in orbit. Then, by changing either the optical properties of the surface of the PW-Sat or the solar cell ratio of the satellite surface, several simulations are presented to illustrate how different configurations satisfy the given temperature limits.

\section{SYSTEM DESCRIPTION AND ASSUMP- TIONS}

The intended use of the model developed in this paper is twofold: 1) to study the effect of different surface compositions (including solar cells) on temperature, 2) to evaluate the possibility of installing a propellant tank in the CubeSat. With regard to the modeling, the following assumptions are made:

\subsection{Structural Assumption}

PW-Sat is a cubic structural bus with a total mass of 1 $\mathrm{kg}$ composed of six faces as walls. The basic structure of these faces is composed of the aluminum alloy 6061-T6 with various optical surface properties. These properties are based on uncoated surfaces for one experiment and coated with a magnesium oxide-aluminum oxide paint for the others. The nitrogen fuel tank, made of stainless steel with a diameter of $5 \mathrm{~cm}$, is planned to be installed in the center of this satellite as shown in Fig. 1a and is assumed to contain an internal gas subject to 100 bars of pressure at an initial temperature of $298 \mathrm{~K}$.

The solar cells will be attached to sides 1,2 and 4 of the PW-Sat, as shown schematically in Fig. 1b, because these faces will be exposed to solar radiation due to the assumed orbit of this satellite.

\subsection{Orbital Assumption}

The PW-Sat is designed for a circular low Earth orbit (LEO). The total orbital period $(P)$ is $1.5 \mathrm{~h}$. However,

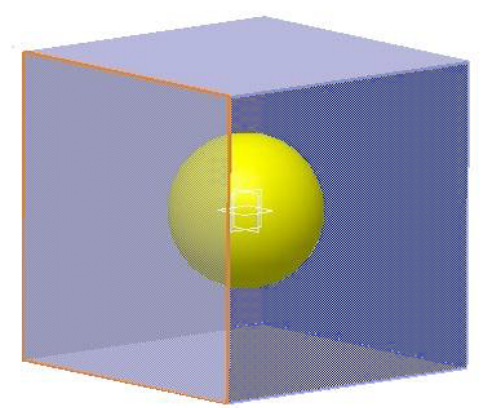

(a)

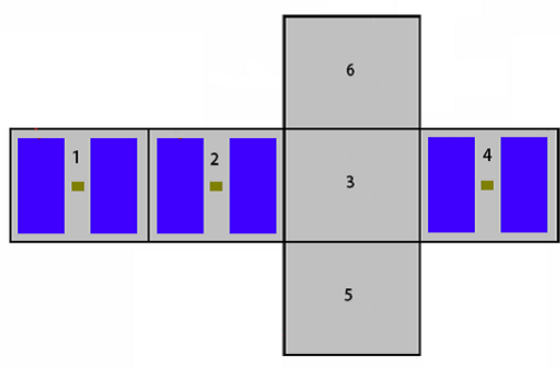

(b)

Figure 1: PW-Sat Structure ((a) PW-Sat spherical fuel tank set, (b) PW-Sat solar cell arrangements).

the motion of PW-Sat is assumed to be identical when exposed to solar radiation and during shadow passage at an altitude of $300 \mathrm{~km}$ and an inclination of zero. Face 3 is directed towards the Earth throughout its orbit. Faces 1, 2 and 4 are exposed to the sun with regard to the orbital motion of the satellite. Finally, faces 5 and 6 are directed towards the space along the satellite orbit as shown in Fig. 2.

\subsection{Thermal Assumption}

The six faces of the PW-Sat are considered to have a uniform temperature distribution. The conductive heat transfer between the fuel tank and satellite is ignored to simplify the thermal modeling calculations. Only face 3 is exposed to infrared radiation from the Earth in this orbit and the albedo during the luminous orbit intervals

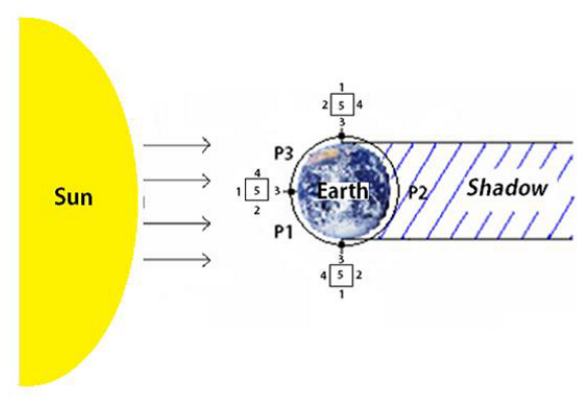

Figure 2: PW-Sat orbital motion. 
$[14,16]$. The thermal rate of power dissipation generated from the operation of elements from the satellite is assumed to be $2 \mathrm{~W}$. The thermal limits of the fuel tank are $228 \mathrm{~K}$ and $338 \mathrm{~K}$, and for the surface area of the satellite are $173 \mathrm{~K}$ and $373 \mathrm{~K}$.

\section{THERMAL MATHEMATICAL MODEL (TMM)}

The problem concerns the formulation of the model, which is on the one hand simple enough to limit the expenditure, on the other hand, detailed enough to present an adequate description of the physical surroundings [17]. The main purpose of these calculations is to divide the periodic motion of the satellite (with period $P$ ) into three intervals (parts) as shown in Fig. 2. The first interval $\left(P_{1}\right)$ starts with an initial time of $t=0 \mathrm{~s}$ when face 1 faces the sun and ends at a time of $t=1350 \mathrm{~s}$ when face 4 faces the sun. The second interval $\left(P_{2}\right)$ is an eclipse interval between $t=1351 \mathrm{~s}$ and $t=4050 \mathrm{~s}$. The third interval $\left(P_{3}\right)$ starts at $t=4051 \mathrm{~s}$ when face 2 faces the sun and ends at the end of the satellite period at $t=5399$ s.

\subsection{First Interval Equations}

$$
\text { Interval } P_{1}: t=0 \rightarrow t=\frac{P}{4}
$$

The satellite spends a quarter of its orbital period in this luminous part. During this time interval, the surface of the satellite receives direct solar and albedo radiation depending on the position of the satellite due to its motion and the satellite emits thermal IR radiation into space; however, only face 3 receives additional infrared radiation from Earth because it faces the Earth [18]. The rate of heat transfer between face 1, the external environment and the spherical fuel tank during the first interval can be described by

$$
\begin{gathered}
\left(m_{\mathrm{Al}} C_{p}+m_{\mathrm{sc}} C_{p}^{\mathrm{sc}}\right) \frac{d T_{1}}{d t}= \\
G_{\mathrm{s}} a_{\mathrm{s}}^{\mathrm{Al}-\mathrm{sc}} A \cos \left(\frac{2 \pi t}{P}\right)+\dot{Q}+\dot{Q}_{F_{1}}-\varepsilon_{\mathrm{IR}}^{\mathrm{Al}-\mathrm{sc}} \sigma A T_{1}^{4}
\end{gathered}
$$

where $m_{\mathrm{Al}}$ denotes the mass of aluminum, $C_{p}$ stands for the specific heat of aluminum $(980 \mathrm{~J} /(\mathrm{kg} \cdot \mathrm{K})), m_{\mathrm{sc}}$ represents the mass of the solar cell , $C_{p}^{\mathrm{sc}}$ is the specific heat of the solar cell $(1600 \mathrm{~J} /(\mathrm{kg} \cdot \mathrm{K})), T_{1}$ denotes the temperature of face $1, G_{\mathrm{S}}$ stands for the solar constant (1367 $\left.\mathrm{W} / \mathrm{m}^{2}\right)$, and $a_{\mathrm{s}}^{\mathrm{Al}-\mathrm{sc}}$ represents the average solar absorptance of aluminum and the solar cell which is calculated as $\left(\mathrm{Al} \% \cdot a_{\mathrm{s}}^{\mathrm{Al}}+\mathrm{sc} \% \cdot a_{\mathrm{s}}^{\mathrm{sc}}\right)$, where $\mathrm{Al} \%$ and sc $\%$ denote the percentages of aluminum and solar cells in the cover, respectively. $A$ stands for the surface area of the face $\left(0.01 \mathrm{~m}^{2}\right), \dot{Q}$ represents the thermal rate of power dissipation, $\dot{Q}_{F_{1}}$ denotes the radiative heat transfer between face 1 and the tank, $\varepsilon_{\mathrm{IR}}^{\mathrm{Al}-\mathrm{sc}}$ is the average infrared emissivity of $\mathrm{Al}$ and sc which is calculated as $\left(\mathrm{Al} \% \cdot \varepsilon_{\mathrm{Al}}+\mathrm{sc}\right.$ $\left.\% \cdot \varepsilon_{\mathrm{sc}}\right)$ and $\sigma$ stands for the Stefan-Boltzmann constant $\left(5.669 \cdot 10^{-8} \mathrm{~W} / \mathrm{m}^{2} \mathrm{~K}^{4}\right)$ [6].

The rate of heat transfer between face 2 , the external environment and the spherical fuel tank during the first interval can be described by

$$
\begin{array}{r}
\left(m_{\mathrm{Al}} C_{p}+m_{\mathrm{sc}} C_{p}^{\mathrm{sc}}\right) \frac{d T_{2}}{d t}= \\
\dot{Q}+\dot{Q}_{F_{2}}-\varepsilon_{I R}^{\mathrm{Al}-\mathrm{sc}} \sigma A T_{2}^{4}
\end{array}
$$

where $T_{2}$ denotes the temperature of face 2 and $\dot{Q}_{F_{2}}$ stands for the radiative heat transfer between face 2 and the tank.

The rate of heat transfer between face 3 , the external environment and spherical fuel tank during the first interval can be described by

$$
\begin{array}{r}
m C_{p} \frac{d T_{3}}{d t}=A F \cdot G_{\mathrm{s}} a_{\mathrm{s}}^{\mathrm{Al}} F_{\mathrm{sE}} A \cos \left(\frac{2 \pi t}{P}\right)+ \\
\dot{Q}+\dot{Q}_{F_{3}}+a_{\mathrm{IR}}^{\mathrm{Al}} \sigma A T_{\mathrm{E}}^{4}-\varepsilon_{\mathrm{IR}}^{\mathrm{Al}} \sigma A T_{3}^{4}
\end{array}
$$

where $m$ denotes the mass of the face $(0.04 \mathrm{~kg}), F_{\mathrm{sE}}$ stands for the view factor between the face of the satellite and the Earth which is almost one [18], $T_{3}$ represents the temperature of face $3, \dot{Q}_{F_{3}}$ is the radiative heat transfer between face 3 and the tank, $A F$ denotes the factor on the albedo $(0.28), a_{\mathrm{s}}^{\mathrm{Al}}$ stands for the solar absorptivity of aluminum, $a_{\mathrm{IR}}^{\mathrm{Al}}$ represents the infrared absorptivity of aluminum, $T_{\mathrm{E}}$ is the reference temperature of the Earth (255 $\mathrm{K})$ and $\varepsilon_{\mathrm{IR}}^{\mathrm{Al}}$ denotes the infrared emissivity of aluminum.

The rate of heat transfer between face 4 , the external environment and the spherical fuel tank during the first interval can be modeled as

$$
\begin{gathered}
\left(m_{\mathrm{Al}} C_{p}+m_{\mathrm{sc}} C_{p}^{\mathrm{sc}}\right) \frac{d T_{4}}{d t}= \\
G_{\mathrm{s}} a_{\mathrm{s}}^{\mathrm{Al}-\mathrm{sc}} A \sin \left(\frac{2 \pi t}{P}\right)+\dot{Q}+\dot{Q}_{F_{4}}-\varepsilon_{\mathrm{IR}}^{\mathrm{Al}} \sigma A T_{4}^{4}
\end{gathered}
$$

where $T_{4}$ denotes the temperature of face 4 and $\dot{Q}_{F_{4}}$ stands for the radiative heat transfer between face 4 and the tank.

The rate of heat transfer between face 5, the external environment and the spherical fuel tank during the first interval can be described as

$$
m C_{p} \frac{d T_{5}}{d t}=\dot{Q}+\dot{Q}_{F_{5}}-\varepsilon_{\mathrm{IR}}^{\mathrm{Al}} \sigma A T_{5}^{4}
$$

where $T_{5}$ denotes the temperature of face 4 and $\dot{Q}_{F_{5}}$ stands for the radiative heat transfer between face 5 and the tank.

The rate of heat transfer between face 6 , the external environment and the spherical fuel tank during the first interval can be described as

$$
m C_{p} \frac{d T_{6}}{d t}=\dot{Q}+\dot{Q}_{F_{6}}-\varepsilon_{\mathrm{IR}}^{\mathrm{Al}} \sigma A T_{6}^{4}
$$

where $T_{6}$ denotes the temperature of face 6 and $\dot{Q}_{F_{6}}$ stands for the radiative heat transfer between face 6 and the tank. 


\subsection{Second Interval Equations}

$$
\text { Interval } P_{2}: t=\frac{P}{4} \rightarrow t=\frac{3}{4} P
$$

These concern the duration of an eclipse. The satellite spends half of its orbital period in an eclipse. During this interval, the surface of the satellite receives neither direct solar nor albedo radiation, whilst face 3 still receives IR radiation from the Earth because it faces it. The satellite emits thermal IR radiation into space. Therefore, the rates of heat transfer of the faces of the satellite (1, 3 and 4) have slightly changed in their equations compared to the first interval.

The rate of heat transfer between faces 1,3 and 4 as well as the external environment during the second interval can be described by the following equations

$$
\begin{gathered}
\left(m_{\mathrm{Al}} C_{p}+m_{\mathrm{sc}} C_{p}^{\mathrm{sc}}\right) \frac{d T_{1}}{d t}=\dot{Q}+\dot{Q}_{F_{1}}-\varepsilon_{\mathrm{IR}}^{\mathrm{Al}-\mathrm{sc}} \sigma A T_{1}^{4} \\
m C_{p} \frac{d T_{3}}{d t}=\dot{Q}+\dot{Q}_{F_{3}}+a_{\mathrm{IR}}^{\mathrm{Al}} \sigma A T_{\mathrm{E}}^{4}-\varepsilon_{\mathrm{IR}}^{\mathrm{Al}} \sigma A T_{3}^{4} \\
\left(m_{\mathrm{Al}} C_{p}+m_{\mathrm{sc}} C_{p}^{\mathrm{sc}}\right) \frac{d T_{4}}{d t}= \\
\dot{Q}+\dot{Q}_{F_{4}}-\varepsilon_{\mathrm{IR}}^{\mathrm{Al}-\mathrm{sc}} \sigma A T_{4}^{4}
\end{gathered}
$$

\subsection{Third Interval Equations}

$$
\text { Interval } P_{3}: t=\frac{3}{4} P \rightarrow t=P
$$

The satellite spends a quarter of its orbital period in this second luminous part. During this time interval, the surface of the satellite receives and emits thermal radiation in a similar manner to during interval 1 with only a slight change in the equation of face 2 .

The rate of heat transfer between this face, the external environment and the spherical fuel tank during the third interval can be modeled as

$$
\begin{gathered}
\left(m_{\mathrm{Al}} C_{p}+m_{\mathrm{sc}} C_{p}^{\mathrm{sc}}\right) \frac{d T_{2}}{d t}= \\
G_{\mathrm{s}} a_{\mathrm{s}}^{\mathrm{Al}-\mathrm{sc}} A\left|\sin \left(\frac{2 \pi t}{P}\right)\right|+ \\
\dot{Q}+\dot{Q}_{F_{2}}-\varepsilon_{I R}^{A l-s c} \sigma A T_{2}^{4}
\end{gathered}
$$

\subsection{The transient heat transfer of the spheri- cal propellant tank}

The rate of heat transfer between the faces of the satellite and the fuel tank can be described by the following equation

$$
\left(m_{\mathrm{s}} C_{p}^{\mathrm{s}}+m_{\mathrm{g}} C_{V}\right) \frac{d T_{\mathrm{t}}}{d t}=-\sum_{1}^{6} \dot{Q}_{F_{n}}
$$

Table 1: Material properties of PW-Sat.

\begin{tabular}{c|ccc}
\hline & $\begin{array}{c}\text { Al 6061-T6 } \\
\text { uncoated }\end{array}$ & $\begin{array}{c}\text { Al 6061-T6 } \\
\text { coated }\end{array}$ & $\begin{array}{c}\text { Solar } \\
\text { cells }\end{array}$ \\
\hline $\begin{array}{c}\text { Specific Heat } \\
{[\mathrm{J} /(\mathrm{kg} \cdot \mathrm{K})]}\end{array}$ & 980 & 980 & 1600 \\
Emissivity (thermal) & 0.08 & 0.92 & 0.85 \\
Absorptivity (solar) & 0.379 & 0.09 & 0.92 \\
\hline
\end{tabular}

Table 2: Material properties of fuel tank.

\begin{tabular}{c|cc}
\hline & Stainless Steel & Nitrogen \\
\hline Specific Heat $[\mathrm{J} /(\mathrm{kg} \cdot \mathrm{K})]$ & 504 & 743 \\
Mass $[\mathrm{kg}]$ & 0.0926 & 0.0074 \\
\hline
\end{tabular}

Table 3: Average of optical surface properties of partially covered surfaces.

\begin{tabular}{c|ccc}
\hline Cube Face & $\varepsilon$ & $\mathrm{a}$ & Coverage \\
\hline \multirow{2}{*}{1,2 and 4} & 0.89 & 0.33 & $70 \% \mathrm{Al}, 30 \% \mathrm{sc}$ \\
& 0.87 & 0.67 & $30 \% \mathrm{Al}, 70 \% \mathrm{sc}$ \\
\hline 3,5 and 6 & 0.92 & 0.09 & $\mathrm{Al}$ painted \\
\hline
\end{tabular}

where $m_{\mathrm{s}}$ denotes the mass of stainless steel, $C_{p}^{\mathrm{s}}$ stands for the specific heat of stainless steel $(504 \mathrm{~J} /(\mathrm{kg} \cdot \mathrm{K})), m_{\mathrm{g}}$ represents the mass and $C_{V}$ the specific heat of nitrogen $(743 \mathrm{~J} /(\mathrm{kg} \cdot \mathrm{K}))$, and $T_{\mathrm{t}}$ is the temperature of the tank.

The radiative heat transfer between each face and the tank $\dot{Q}_{F_{n}}$, depending on which face it applies to, can be described as

$$
\dot{Q}_{F_{n}}=F_{\mathrm{ft}} \varepsilon \sigma A\left(T_{\mathrm{t}}^{4}-T_{n}^{4}\right)
$$

The view factor between the face in question and the fuel tank is given by $F_{\mathrm{ft}}=\frac{1}{(1+H)^{2}}$ (see, e.g. $\left.[10,11]\right)$, where $H$ denotes the ratio of the distance between the spherical surface of the tank to the surface of the internal face ( $h=$ $0.025 \mathrm{~m})$ in terms of the radius $(r=0.025 \mathrm{~m})$, which is expressed as $H=\frac{h}{r}$.

The mass of the solar cell $m_{\mathrm{sc}}$ per unit area is on average $850 \mathrm{~g} / \mathrm{m}^{2}$, thus, the mass of the solar cell as a proportion of the total mass of the face is determined by the equation $\left(m_{\mathrm{sc}}=850 \mathrm{~g} / \mathrm{m}^{2} \cdot A \cdot \mathrm{sc} \%\right)$. The total mass of the tank $m_{\mathrm{t}}$ is assumed to be $0.1 \mathrm{~kg}$, so the mass of nitrogen gas was calculated by assuming the initial temperature and total pressure of the tank. The optical surface properties are shown in Table 1, and the masses of both nitrogen gas and the tank are shown in Table 2. Hence, it is necessary to calculate the properties of the average materials to conduct a thermal analysis. The emissivity of infrared radiation from these faces can be calculated as the average of the emissivity of infrared radiation from aluminum and the emissivity of infrared radiation from the solar cell in addition to the absorptivity of these faces as shown in Table 3. 


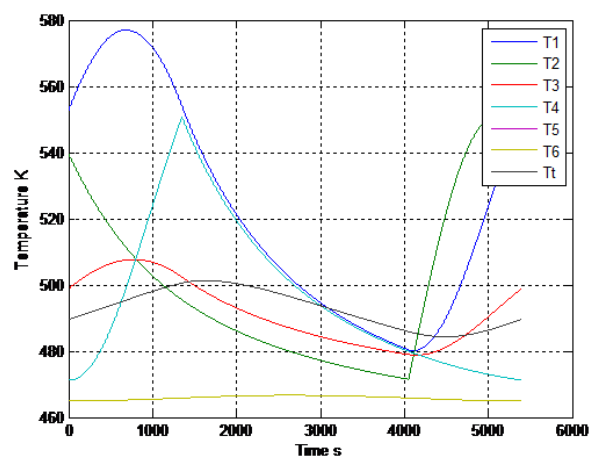

(a)

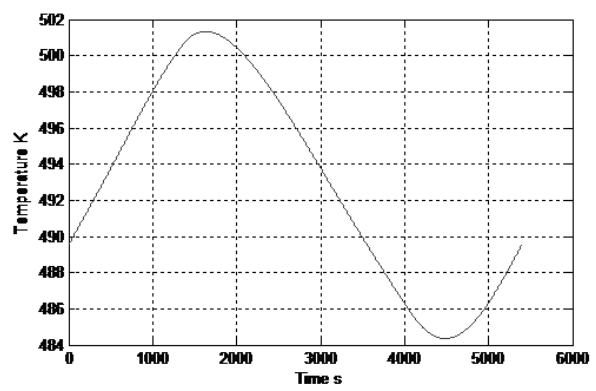

(b)

Figure 3: The thermal behavior using $100 \% \mathrm{Al}$ during one orbital period ((a) temperatures of the faces and fuel tank, (b) temperature of the fuel tank) (T1, ., T6) refers to the temperatures of faces $1, \ldots, 6$, respectively, and $\left(T_{t}\right)$ denotes the temperature of the tank.

\section{COMPUTATIONAL RESULTS}

In this section, the faces and investigations into the thermal behavior of the tank are presented for several cases based on: uncoated surfaces, surfaces coated with magnesium oxide-aluminum oxide paint and different feasible options of the ratios of solar cells from the PW-Sat to simulate the temperature of the fuel tank with different optical properties of the surface materials and solar cell ratios.

\subsection{The PW-Sat faces composed of $100 \%$ uncoated aluminum}

In this case, the thermal simulations of the faces and fuel tank were conducted according to the assumption that the faces of the satellite are composed of the aluminum alloy 6061-T6. Starting with the equations of the intervals and by using the ODE45 solver in MATLAB (the simulation time step was $1 \mathrm{~s}$ ), the thermal behavior during the orbital motion of the satellite was computed.

1 - The thermal simulation of the faces and spherical fuel tank during one orbital period (time span from $0 \mathrm{~s}$ to $5399 \mathrm{~s}$ ) is shown in Fig. 3a and the simulation of the temperature of the tank during this orbital period is shown in Fig. 3b. It can be seen that the predefined temperature limits are not adhered to in this case, since the minimum

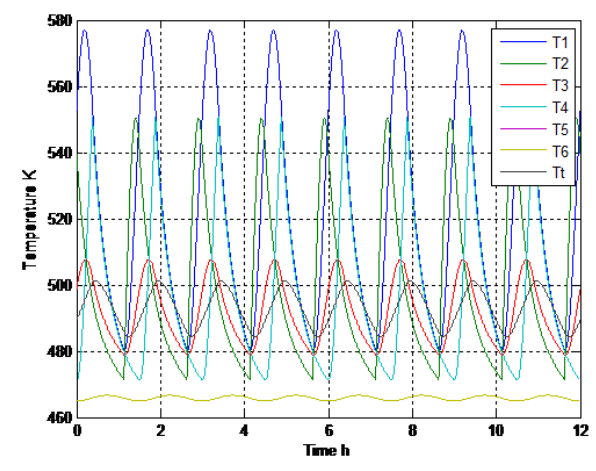

(a)

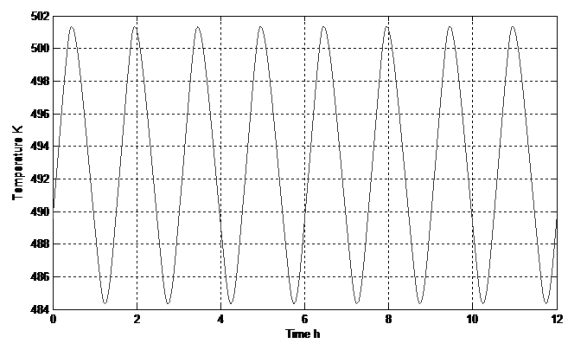

(b)

Figure 4: Thermal behaviors using $100 \% \mathrm{Al}$ during 8 orbital periods ((a) temperatures of the faces and fuel tank, (b) temperature of the fuel tank).

temperatures of the faces of the satellite and fuel tank exceed $460 \mathrm{~K}$ during its orbital period.

2 - The thermal simulation of the faces and spherical fuel tank during several orbits ( 8 orbital periods with a time span of $12 \mathrm{~h}$ to illustrate long-term operations) is shown in Fig. 4a, and the simulation of the temperature of the tank during these orbital periods is shown in Fig. $4 b$.

\subsection{The faces of the PW-Sat are composed of $100 \%$-coated aluminum}

The thermal simulations of the faces and fuel tank were conducted according to the assumption that the surface of the satellite was composed of aluminum coated with magnesium oxide-aluminum oxide paint. By using the assumed time span of each interval, the results are shown below:

1 - The thermal simulations of the faces and spherical fuel tank during one orbital period (time span from $0 \mathrm{~s}$ to $5399 \mathrm{~s}$ ) are shown in Fig. 5a, and the simulation of the temperature of the tank during this orbital period is shown in Fig. 5b. It can be seen that all the defined temperature limits are adhered to in this case.

2 - The thermal simulation of the faces and spherical fuel tank over 8 orbital periods (time span of $12 \mathrm{~h}$ ) is shown in Fig. 6a, and the simulation of the temperature of the tank during these orbital periods is shown in Fig. $6 b$. 


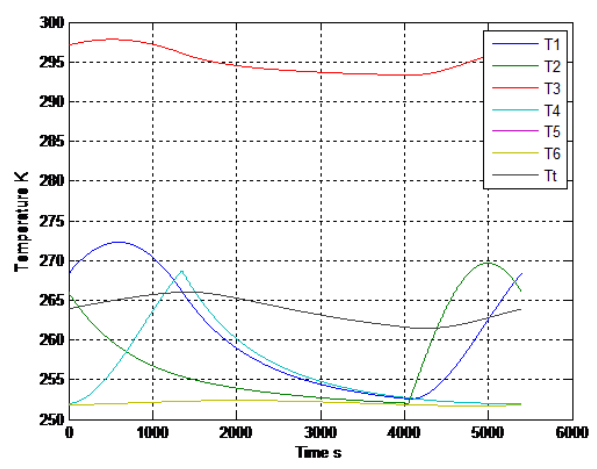

(a)

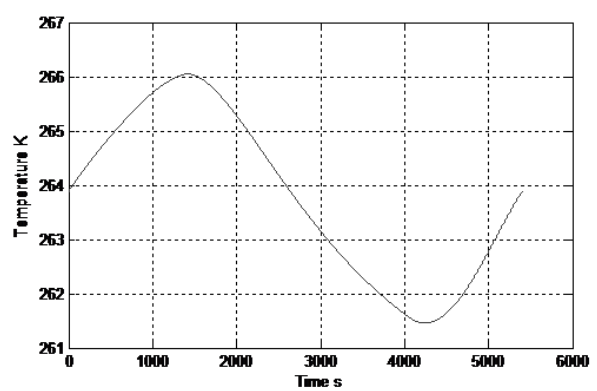

(b)

Figure 5: Thermal behaviors using coated Al during one orbital period ((a) temperatures of the faces and fuel tank, (b) temperature of the fuel tank).

\subsection{The faces of the satellite exposed to the sun during its orbit are covered with $70 \%$ aluminum and $30 \%$ solar cells}

In this case, these three sides of the PW-Sat are assumed to be composed of $70 \%$ aluminum and $30 \%$ solar cells and the other faces are coated with magnesium oxidealuminum oxide paint. The simulation results are as follows:

1 - The thermal simulation of the faces and spherical fuel tank during one orbital period (time span of $0 \mathrm{~s}$ to $5399 \mathrm{~s}$ ) is shown in Fig. 7a and the simulation of the temperature of the tank during this orbital period is also shown separately in Fig. 7b. The results show that the temperature of the tank varied between a maximum of $281.9 \mathrm{~K}$ and a minimum of $265.4 \mathrm{~K}$, while the temperatures of the faces also remained within the given temperature limits.

2 - The thermal simulation of the faces and spherical fuel tank over 8 orbital periods (time span of $12 \mathrm{~h}$ ) is shown in Fig. 8a, and the simulation of the temperature of the tank during these orbital periods is shown in Fig. $8 b$.

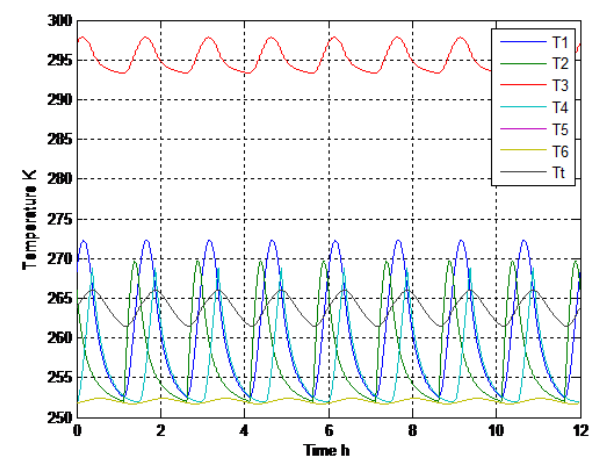

(a)

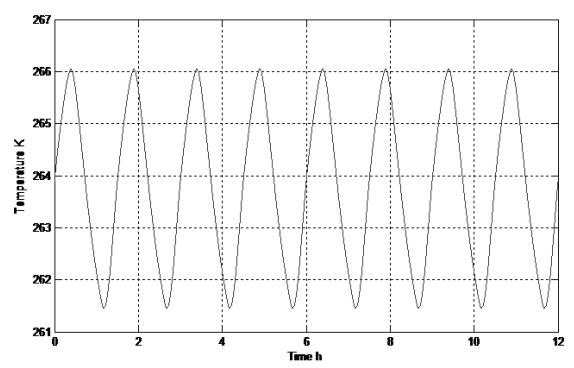

(b)

Figure 6: Thermal behaviors using coated $\mathrm{Al}$ over 8 orbital periods ((a) temperatures of the faces and fuel tank, (b) temperature of the fuel tank).

\subsection{The faces of the satellite exposed to the sun during its orbit are covered with $30 \%$ aluminum and $70 \%$ solar cells}

The results, according to the assumption that three sides of the PW-Sat are composed of $30 \%$ aluminum and 70 $\%$ solar cells while the rest of them are coated with magnesium oxide-aluminum oxide paint, are shown below:

1 - The thermal simulation of the faces and spherical fuel tank during one orbital period (time span from $0 \mathrm{~s}$ to 5399 s) is shown in Fig. 9a, while simulation of the temperature of the tank during this orbital period is shown in Fig. 9b. The results show that the temperature of the tank varied between a maximum of $302.4 \mathrm{~K}$ and a minimum of $270.6 \mathrm{~K}$, while the temperatures of the faces were within thermal limits.

2 - The thermal simulation of the faces and spherical fuel tank over 8 orbital periods (time span of $12 \mathrm{~h}$ ) is shown in Fig. 10a, while the simulation of the temperature of the tank during these orbital periods is shown in Fig. 10b.

\section{Conclusion}

A thermal mathematical model was constructed and studied to compute the temperatures of the surfaces and fuel tank of the PW-Sat. Several cases were presented using various surface compositions and the results show that the proposed TMM is able to calculate the radiative heat 


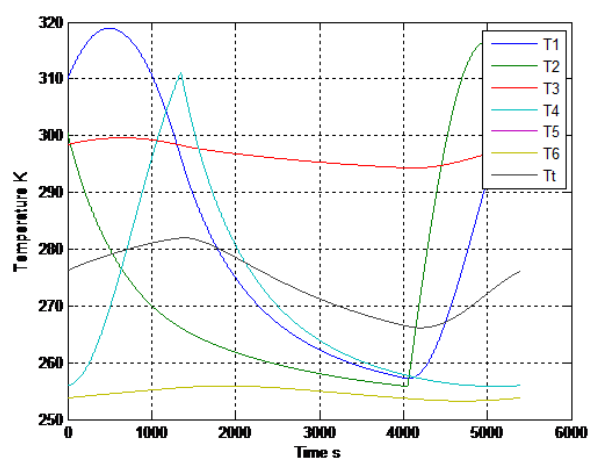

(a)

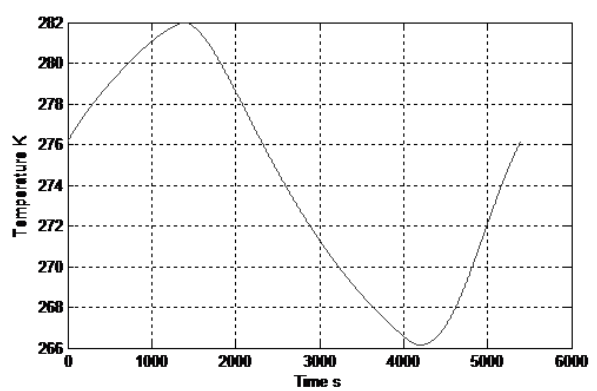

(b)

Figure 7: Thermal behaviors with $30 \%$ sc during one orbital period ((a) temperatures of the faces and fuel tank, (b) temperature of the fuel tank).

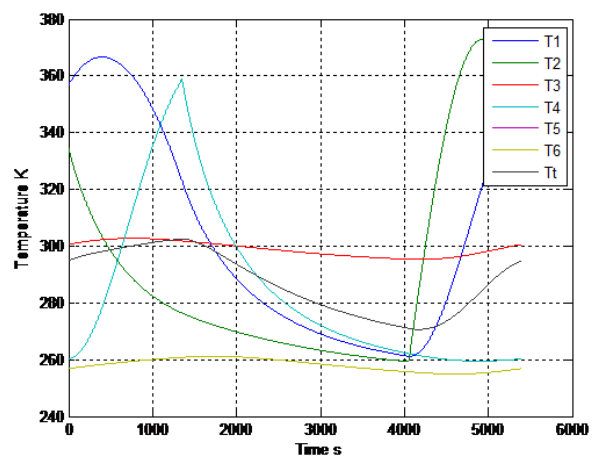

(a)

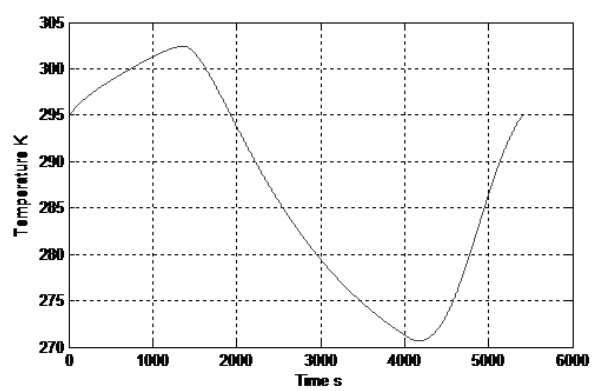

(b)

Figure 9: Thermal behaviors with $70 \%$ sc during one orbital period ((a) temperatures of the faces and fuel tank,(b) temperature of the fuel tank).

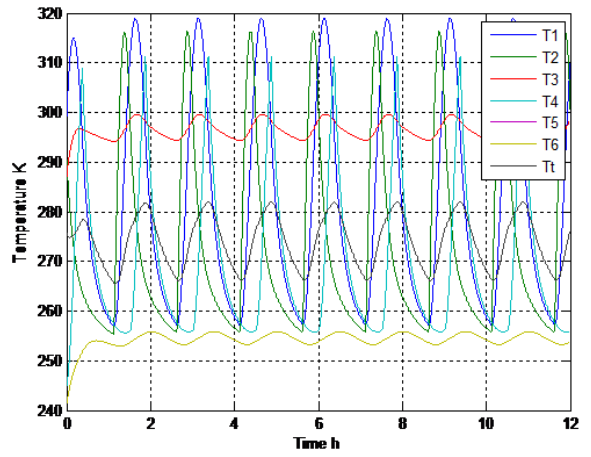

(a)

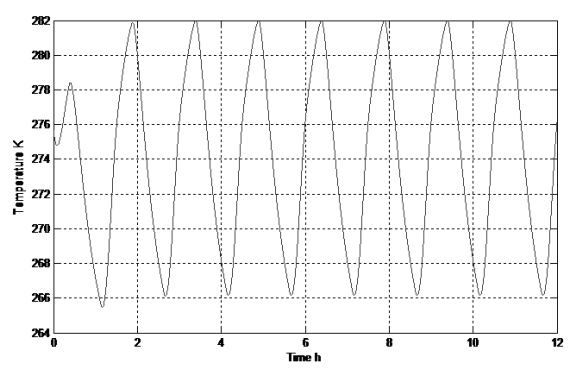

(b)

Figure 8: Thermal behaviors with $30 \%$ sc over 8 orbital periods ((a) temperatures of the faces and fuel tank, (b) temperature of the fuel tank).

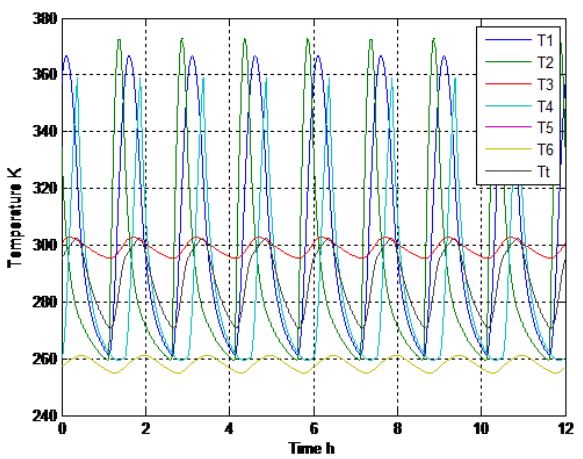

(a)

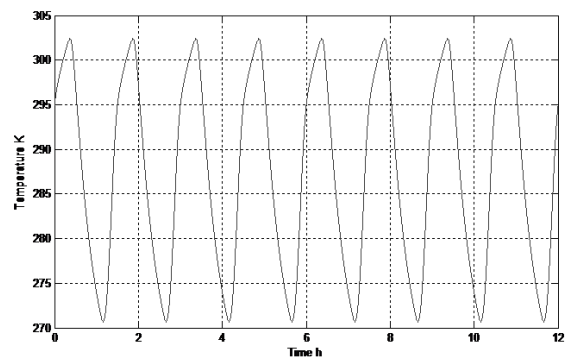

(b)

Figure 10: Thermal behaviors with $70 \%$ sc over 8 orbital periods ((a) temperatures of the faces and fuel tank, (b) temperature of the fuel tank). 
that the PW-Sat would encounter during its assumed orbit. Initially, the surfaces of the PW-Sat were assumed to be composed of $100 \%$ of the aluminum alloy 6061-T6. The corresponding results suggest that the temperatures of the surfaces and fuel tank would be too high. Therefore, additional finishes applied to the surfaces were taken into consideration. The first choice of finish was to coat the entire surface of the satellite with magnesium oxidealuminum oxide paint. The obtained results show that the temperatures of the surfaces and fuel tank dropped because of the increasing emissivity and decreasing absorptivity of the surfaces. Further simulations were performed of cases in which the faces were exposed to the sun when partially covered with solar cells. The results indicate that the case described in Subsection 4.4, which delivers the most electrical power due to the highest percentage of solar cells, still satisfies the temperature limits of the fuel tank and surfaces of the satellite. The results also suggest that it is possible to install a fuel tank inside the PW-Sat which could be the first step required to add a propulsion system that can generate thrust for this CubeSat. Future works will include validation of the model using advanced thermal simulation tools as well as active control design to precisely regulate the temperature of the fuel tank.

\section{Acknowledgement}

This work has been partially supported by the European Union, co-financed by the European Social Fund through the grant EFOP-3.6.3-VEKOP-16-2017-00002. The support of the University of Technology of Baghdad and Warsaw University of Technology is also acknowledged.

\section{REFERENCES}

[1] Tummala, A. R.; Dutta, A.: An overview of cube-satellite propulsion technology and trends, Aerospace, 2017 4(4), 58. DOI: 10.3390/aerospace4040058

[2] Mehrparvar, A.: CubeSat design specification Rev. 13, The CubeSat Program, Cal Poly SLO, 2015. http://www. cubesat.org/resources

[3] PW-Sat description (CubeSat Warsaw University of Technology). https://directory.eoportal.org/web/ eoportal/satellite-missions/p/pw-sat

[4] Lemmer, K.: Propulsion for CubeSats, Acta Astronaut., 2017 134, 231-243. DOI: 10.1016/j.actaastro.2017.01.048

[5] Gaite, J.: Nonlinear analysis of spacecraft thermal models, Nonlinear Dyn. 2011 65, 283-300. DOI: 10.1007/s11071-010-9890-4
[6] Sutton, G. P.; Biblarz, O.: Rocket propulsion elements, Seventh Edition (Wiley, New York, USA) 2001. ISBN 0-471-32642-9

[7] Mueller, J.; Hofer, R.; Parker, M.; Ziemer, J.: Survey of propulsion options for CubeSats, 57th JANNAF Propulsion Meeting (Colorado) 2010 JANNAF1425 1-56. https://trs.jpl.nasa.gov/bitstream/ handle/2014/41627/10-1646.pdf

[8] Isidori, A.: Nonlinear Control Systems (Springer, London, UK) 1999. ISBN 978-1-84628-615-5

[9] Hangos, K. M.; Bokor, J.; Szederkényi, G.: Analysis and Control of Nonlinear Process Systems (Springer, London, UK) 2004. ISBN 978-1-85233-861-9

[10] Modest, M. F.: Radiative Heat Transfer, Third Edition (Academic Press, Oxford, UK) 2013. ISBN 9780123869906

[11] Sala, A.: Radiation Heat Transfer (in polish) (WNT, Scientific and Technical Publications, Warsaw, Poland) 1982. ISBN 8320403677

[12] Gilmore, D. G.: Spacecraft thermal control handbook, Second Edition, vol. 1. (The Aerospace Press, El Segundo, USA) 2002. ISBN 1-884989-11-X

[13] Diaz-Aguado, M. F.; Greenbaum, J.; Fowler, W. T.; Lightsey, E. G.: Small satellite thermal design, test, and analysis, Modeling, Simulation, and Verification of Space-based Systems III, Proc. SPIE, 2006 6221, 622109. DOI: 10.1117/12.666177

[14] Grande, I. P.; Andres, A. S.; Guerra, C.; Alonso, G.: Analytical study of the thermal behaviour and stability of a small satellite, Appl. Therm. Eng., 2009 29(11-12), 2567-2573. DOI: 10.1016/j.applthermaleng.2008.12.038

[15] Farrahi, A.; Pérez-Grande, I.: Simplified analysis of the thermal behavior of a spinning satellite flying over Sun-synchronous orbits, Appl. Therm. Eng. 2017 125, 1146-1156. DOI: 10.1016/j.applthermaleng.2017.07.033

[16] JAXA: Design standard, Spacecraft thermal control system, JERG-2-310 (Japan Aerospace Exploration Agency, Tsukuba, Japan) 2009. http: //sma . jaxa. jp/ en/TechDoc/Docs/E_JAXA-JERG-2-310_08_RE.pdf

[17] Fortoscue, P.; Stark, J.; Swinerd, G. (eds): Spacecraft systems engineering, Fourth Edition (Wiley, Chichester, UK) 2011. ISBN 978-0-470-75012-4

[18] VanOutryve, C. B.: A thermal analysis and design tool for small spacecraft, Master's Thesis, 2008. https://scholarworks.sjsu.edu/etd_theses/3619 